\title{
Loss of genes for DNA recombination and repair in the reductive genome evolution of thioautotrophic symbionts of Calyptogena clams
}

\author{
Hirokazu Kuwahara ${ }^{1,2}$, Yoshihiro Takaki', Shigeru Shimamura', Takao Yoshida', Taro Maeda ${ }^{3}$, Takekazu Kunieda² \\ and Tadashi Maruyama ${ }^{{ }^{*}}$
}

\begin{abstract}
Background: Two Calyptogena clam intracellular obligate symbionts, Ca. Vesicomyosocius okutanii (Vok; C. okutanii symbiont) and Ca. Ruthia magnifica (Rma; C. magnifica symbiont), have small genomes (1.02 and $1.16 \mathrm{Mb}$, respectively) with low $\mathrm{G}+\mathrm{C}$ contents (31.6\% and $34.0 \%$, respectively) and are thought to be in an ongoing stage of reductive genome evolution (RGE). They lack recA and some genes for DNA repair, including mutY. The loss of recA and mutY is thought to contribute to the stabilization of their genome architectures and GC bias, respectively. To understand how these genes were lost from the symbiont genomes, we surveyed these genes in the genomes from 10 other Calyptogena clam symbionts using the polymerase chain reaction (PCR).
\end{abstract}

Results: Phylogenetic trees reconstructed using concatenated 165 and $23 S$ rRNA gene sequences showed that the symbionts formed two clades, clade I (symbionts of C. kawamurai, C. laubieri, C. kilmeri, C. okutanii and C. soyoae) and clade II (those of C. pacifica, C. fausta, C. nautilei, C. stearnsii, C. magnifica, C. fossajaponica and C. phaseoliformis). recA was detected by PCR with consensus primers for recA in the symbiont of $C$. phaseoliformis. A detailed homology search revealed a remnant recA in the Rma genome. Using PCR with a newly designed primer set, intact recA or its remnant was detected in clade II symbionts. In clade I symbionts, the recA coding region was found to be mostly deleted.

In the Rma genome, a pseudogene of mutY was found. Using PCR with newly designed primer sets, mutY was not found in clade I symbionts but was found in clade II symbionts. The G+C content of $16 S$ and 23S rRNA genes in symbionts lacking mutY was significantly lower than in those with mutY.

Conclusions: The extant Calyptogena clam symbionts in clade II were shown to have recA and mutY or their remnants, while those in clade I did not. The present results indicate that the extant symbionts are losing these genes in RGE, and that the loss of mutY contributed to the GC bias of the genomes during their evolution.

\section{Background}

Recent genome analyses have shown that the genomes of intracellular obligate symbionts, which are vertically transmitted to the next generation of their hosts, have a tendency to reduce in size during evolution [1-3]. Generally, smaller genomes have a lower $\mathrm{G}+\mathrm{C}$ content (GC bias) [3], although there are some exceptions, e.g., a symbiont of cicadas, $\mathrm{Ca}$. Hodgkinia cicadicola has a very

\footnotetext{
* Correspondence: tadashim@jamstec.go.jp

${ }^{1}$ Marine Biodiversity Research Program, Japan Agency for Marine-Earth Science and Technology, Natsushima-cho, Yokosuka, Kanagawa 237-0061, Japan

Full list of author information is available at the end of the article
}

small genome $(150 \mathrm{~kb})$ with a relatively high $\mathrm{G}+\mathrm{C}$ content (58.4\%) [4]. Genes for DNA recombination and repair, i.e., rec $A$ and $u v r A$ to $C$, are deleted in many intracellular symbiont genomes $[1,5,6]$.

Reductive genome evolution (RGE) has been extensively studied in insect symbionts. Buchnera strains, which are intracellular heterotrophic symbionts of aphids, have small genomes (0.45-0.65 Mb). Although the genome architectures of the extant Buchnera strains are stable, RGE is ongoing with small deletions at a slower rate than in its early stage [7-9]. However, the earlier stages of RGE are still largely unknown.

\section{Ciomed Central}


Intracellular symbiotic chemoautotrophic bacteria are ubiquitous in deep-sea invertebrates such as Calyptogena clams [10]. Calyptogena clam symbionts are thought to be vertically transmitted via eggs $[11,12]$. The similarity between the phylogenetic topologies of Calyptogena clams and their symbionts indicates their co-evolution [13], although the possibility of lateral acquisition of the symbionts in some Calyptogena clams has been reported $[14,15]$. The genomes of symbionts in Calyptogena magnifica (Ca. Ruthia magnifica, Rma, 1.16 $\mathrm{Mb}$ ) and in C. okutanii ( $\mathrm{Ca}$. Vesicomyosocius okutanii, Vok, $1.02 \mathrm{Mb}$ ) have been reported [16,17]. They lack large-sized repeated sequences (> $200 \mathrm{bp})$, phage and mobile genetic elements [16,17]. A comparative analysis of these genomes showed that the RGE in Calyptogena symbiont genomes is currently ongoing and is still in an earlier stage than that in the Buchnera strains [8]. Further, it has been reported that both of the Calyptogena symbionts lack genes for DNA recombination and repair such as recA and mut $Y$ [17].

Recombinase RecA is a key enzyme for homologous recombination [18]. It requires relatively long repeated sequences (> $200 \mathrm{bp}$ ) for recombination [19] and is a possible driving mechanism of dynamic genome rearrangement including large deletions. In RGE in symbionts of Calyptogena clams, RecA probably functioned to delete large gene sequences by recombination, consuming the long repeated sequences in the early stage of RGE [8]. On the other hand, MutY is known to repair A-G mispairs to C-G pairs [20]. Thus, the loss of mutY is thought to cause decreasing $\mathrm{G}+\mathrm{C}$ content of the genome.

While losses of genes for DNA repair and recombination may occur spontaneously, they affect the later stage of RGE by increasing mutation rates, affecting the GC bias and regenerating short repeated sequences [8]. After the loss of $r e c A$, the genome architecture probably stabilized in the clam symbionts [8]. In the insect symbiont Buchnera, recA was reported to be lost in its early evolution [21]. The contribution of RecA to RGE in intracellular symbionts is still controversial. It was shown that small deletions with a size of up to $200 \mathrm{~kb}$ occur without recognizable repeats via RecA-independent recombination events in Salmonella [22]. To understand the roles of RecA-dependent and -independent recombination events in RGE in intracellular symbiosis, it is important to determine when and how recA was lost in their lineages and the effects of its loss on their RGE. However, little is known about the relationship between the loss of DNA repair/recombination genes and RGE. To understand the effects of their loss on RGE, we posed the question of whether these genes had been lost before the divergence of the Calyptogena clam symbionts or whether they remained in some symbionts thereafter. To address this question, we searched for recA, mut $Y$ and/or their remnants in the genomes of 10 Calyptogena clam symbionts in addition to Rma and Vok.

\section{Results}

Phylogenetic relationships of Calyptogena clam symbionts Before screening the genes for DNA recombination and repair, we analyzed the phylogenetic relationships of 12 symbionts of Calyptogena clams (Table 1). Because the resolution of the $16 \mathrm{~S}$ rRNA tree was too low, we amplified and sequenced the 23S rRNA genes (Tables 2 and 3 ) from the symbionts and reconstructed trees using the concatenated 16S and 23S rRNA gene sequences (Figure $1)$. Two clades well supported with high bootstrap values and posterior probabilities were identified. Symbionts of C. kawamurai, C. laubieri, C. kilmeri, C. soyoae and C. okutanii (Vok) formed clade I, and those of C. pacifica, C. fausta, C. nautilei, C. stearnsii, C. magnifica (Rma), C. fossajaponica and C. phaseoliformis formed clade II (Figure 1). The bootstrap support for clade II [bootstrap values at the node in the maximum likelihood $(\mathrm{ML})$ tree $>89$, posterior probability $=1.00$ ] was slightly less than that for clade I (bootstrap values = 100 , posterior probability $=1.00)$. In clade II, the symbionts of $C$. phaseoliformis and $C$. fossajaponica formed a subclade with Rma, and the symbionts of $C$. stearnsii, C. nautilei, C. fausta and C. pacifica formed another subclade with a high bootstrap value (Figure 1). It is noteworthy that the branch length from the node of clade II (** in Figure 1) to Rma was longer than those of other branches in clade II, and that the branch length from the node of the radiation of the Calyptogena clam symbiont (* in Figure 1) to the node of clade I radiation $(* * *$ in Figure 1) was longer than that to clade II $(* *$ in Figure 1). In clade I, two subclades respectively containing symbionts of C. kilmeri, C. soyoae and C. okutanii (Vok) and those of C. kawamurai and C. laubieri were not robust (Figure 1).

\section{DNA recombinase gene, recA, in Calyptogena clam symbionts}

Using PCR with an internal primer set designed from the conserved sequences of recAs of several gamma-proteobacteria (Table 2), we obtained an amplicon from the genomic DNA of a C. phaseoliformis symbiont (data not shown). The sequence obtained was shown to be a part of recA by a BLASTX search in the DDBJ/GenBank/ EMBL. A search for a residual $r e c A$ in the Rma and Vok genomes using this sequence and the Escherichia coli recA sequence revealed that the Rma genome contains a DNA fragment similar to recA between Rmag_0799 (ABC transporter related, Figure 2A) and Rmag_0800 [diaminohydroxyphosphoribosylaminopyrimidine 
Table 1 Calyptogena clams used in the present study

\begin{tabular}{|c|c|c|c|c|c|c|}
\hline Host clam & Date of collection & Dive \# & Depth (m) & Latitude & Longitude & Collection site \\
\hline Calyptogena phaseoliformis & 2006.6 .3 & 6 K\#953 & 6264 & $40.10 \mathrm{~N}$ & 144.19E & Japan Trench \\
\hline Calyptogena fossajaponica & 2006.6 .2 & 6K\#952 & 6182 & $40.10 \mathrm{~N}$ & 144.17E & Japan Trench \\
\hline Calyptogena stearnsii & 1996.3.29 & ROV Biv-0099-0103 & $659-683.5$ & $36.77 \mathrm{~N}$ & $122.04 \mathrm{~W}$ & Monterey Bay \\
\hline Calyptogena nautilei & 2005.6.18 & $6 K \# 884$ & 3306 & $32.58 \mathrm{~N}$ & 134.69E & Okinawa-Nankai Trough \\
\hline Calyptogena pacifica & 1996.3.29 & ROV Biv-0105-0112 & $659-683.5$ & $36.77 \mathrm{~N}$ & $122.04 \mathrm{~W}$ & Monterey Bay \\
\hline Calyptogena fausta & 1996.6.10 & $2 K \# 869$ & 1490 & $34.91 \mathrm{~N}$ & 138.66E & Suruga Bay \\
\hline Calyptogena kawamurai & 2005.6.13 & 6K\#881 & 608 & $34.08 \mathrm{~N}$ & 137.79E & Okinawa-Nankai Trough \\
\hline Calyptogena laubieri & 1997.8.23 & $10 K \# 45$ & 3761 & $33.65 \mathrm{~N}$ & 137.91E & Okinawa-Nankai Trough \\
\hline Calyptogena okutanii & From database* & & & & & \\
\hline Calyptogena kilmeri & 1996.3.25 & ROV Biv-0116-0121 & 900 & $36.73 \mathrm{~N}$ & $122.00 \mathrm{~W}$ & Monterey Bay \\
\hline Calyptogena soyoae & 2000.7.1 & HPD\#13 & 1178 & $35.00 \mathrm{~N}$ & 139.23E & Sagami Bay off Hatsushima \\
\hline Calyptogena magnifica & From database*** & & & & & \\
\hline
\end{tabular}

HPD, Hyper Dolphin; 2K, Shinkai 2000; 6K, Shinkai 6500; ROV, remote operated vehicle; 10K, ROV Kaiko.

*Accession number AP009247.

**Accession number CP000488.

deaminase/5-amino-6-(5-phosphoribosylamino) uracil reductase, Figure 2A], but the Vok genome does not. The entire $r e c A$ sequence and its flanking sequences from the $C$. phaseoliformis symbiont were amplified in PCR using the primers recA_F and recA_R (Table 2), which were designed from the franking conserved sequences of the recA-like sequence in Rma (Rmag 0799 and Rmag 0800) and their respective corresponding sequences in Vok [COSY_0725 (ABC transporter ATPbinding protein) and COSY_0726 (bifunctional riboflavin biosynthesis protein RibD)]. While Rmag_0800 and COSY_0726 are annotated with different gene names, they are homologous to each other.

In PCR using the primers recA_F and recA_R, amplicons with different lengths were obtained from 10 symbionts and sequenced (Table 3). These amplicons were designated as recA-amplicons. In open reading frame (ORF) analysis, we detected intact recA coding for 344amino acid RecA in the symbiont genomes of C. pacifica and C. fossajaponica as well as C. phaseoliformis
(Figure 2A). Their amino acid sequence identities to that of $E$. coli were $70.0 \%, 70.6 \%$ and $70.3 \%$, respectively. However, Rma and the symbionts of C. stearnsii, $C$. fausta and C. nautilei were shown to have defective recAs. In Rma, highly degraded remnants of the $\operatorname{rec} A$ gene were detected (Figure 2A). In the symbionts of $C$. fausta and $C$. nautilei, the $\operatorname{rec} A$ was found to be degraded into a few apparent ORFs (Additional file 1 Figure S1). In both of the symbionts, the 52nd codon "GGT" was replaced with a stop codon "TAG." This was found to be caused by the same insertion of "CC" at the 151st-152nd base from the initiation codon of the original $r e c A$ sequences (at the position between 483 and 484 in Additional file 1 Figure S1).

In the symbiont of $C$. stearnsii, the coding region of recA was found to be fragmented by many stop codons, which were caused by substitutions and a few base insertions, i.e., the substitution of " $\mathrm{C}$ " with " $\mathrm{T}$ " makes the stop "TGA" at the 343rd (10th base from the initiation site in C. phaseoliformis recA) and the insertion of

Table 2 Primers for PCR

\begin{tabular}{|c|c|c|c|c|}
\hline Primer & Nucleotide sequence & Target gene & Coding amino acid sequence & Note \\
\hline recA Fi & 5'-GAYGCNGARCAYGCIYTNGAYCC-3' & recA & DAEHALD & ip \\
\hline recA Ri & 5'-ACNCCDATYTTCATNCKDATYTG-3' & recA & QIRMKIGV & ip \\
\hline recA_F & 5'-GATTGCATATCATTCATCTGATAACG-3' & recA & non & ep \\
\hline recA_R & 5'-AGTGGATTRGGATCAAGCATAGC-3' & $\operatorname{rec} A$ & non & ep \\
\hline mutY_F & 5'-CCTATATGWACACCATCAAAGTTGCC-3' & mutY & non & ep \\
\hline mutY_R & 5'-AATACTTGGRGWAACGCCACTAATG-3' & mutY & non & ep \\
\hline $27 \mathrm{~F}$ & 5'-AGAGTTTGATCCTGGCTCAG-3' & $16 S$ & non & ip \\
\hline $1492 R$ & 5'-GGTTACCTTGTTACGACTT-3' & $16 S$ & non & ip \\
\hline $23 S F 1$ & 5'-GGGAACTGAAACATCTAAGTACC-3' & $23 S$ & non & ip \\
\hline $23 S \mathrm{R} 1$ & 5'-CCCGCTTAGATGCTTTCAG-3' & $23 S$ & non & ip \\
\hline
\end{tabular}

ip, internal primer; ep, external primer.

$16 \mathrm{~S}$, small subunit rRNA gene; $23 \mathrm{~S}$, large subunit rRNA gene. 
Table 3 Accession numbers of symbiont DNA sequences determined in the present study or retrieved from databases

\begin{tabular}{|c|c|c|c|c|}
\hline Host clam (Abbreviation of symbiont) & recA amplicon ${ }^{\#}$ & mutY amplicon \$ & 16S rDNA & $23 \mathrm{~S}$ rDNA \\
\hline Bathymodiolus septemdierum (Bsep S) & n.d. & n.d. & AB598130 & AB598131 \\
\hline Calyptogena phaseoliformis (Cpha S) & AB586104 & AB586113 & AB479082* & AB598132 \\
\hline C. fossajaponica (Cfos S) & AB586105 & AB586114 & $\mathrm{AB} 044744^{*}$ & AB598133 \\
\hline C. stearnsii (Cste S) & AB642236 & AB642237 & AB642238 & AB642239 \\
\hline C. nautilei (Cnau S) & AB586106 & AB586115 & AB479080* & AB598134 \\
\hline C. pacifica (Cpac S) & AB586107 & AB586116 & AF03572* & AB598135 \\
\hline C. fausta (Cfau S) & AB586108 & AB586117 & AB47908* & AB598137 \\
\hline C. kawamurai (Ckaw S) & AB586109 & AB586118 & AB479076* & AB598138 \\
\hline C. laubieri (Clau S) & AB586110 & AB586119 & $\mathrm{AB} 073121^{*}$ & AB598139 \\
\hline C. okutanii (Vok) & AP009247* & AP009247* & AP009247* & AP009247* \\
\hline C. kilmeri (Ckil S) & AB586111 & AB586120 & AF035720* & AB598136 \\
\hline C. soyoae (Csoy S) & AB586112 & AB586121 & AB479077* & AB598140 \\
\hline C. magnifica (Rma) & CP000488* & СР000488* & СР000488* & CP000488* \\
\hline
\end{tabular}

\#Gene region amplified with primers recA_F and recA_R.

\$Gene region amplified with primers mutY_F and mutY_R.

n.d., Not determined in the present study.

*Not determined in the present study but retrieved from a database.

"A" between the 427th and 428th base makes the stop "TAA" at 438-440 in Additional file 1 Figure S1 (Figures $2 \mathrm{~A}$ and Additional file 1 Figure S1). No common insertion, deletion or substitutional mutation was detected between the symbiont of $C$. stearnsii and those of $C$. nautilei and C. fausta. In the symbionts of C. pacifica, C. fossajaponica, C. phaseoliformis, C. fausta and C. nautilei, an apparently intact ORF coding $r e c X$ was found downstream of recA (Figure 2A). However, the symbiont of $C$. stearnsii was found to contain a pseudogene of $\operatorname{rec} X$ (Figure 2A).

We performed multiple alignments of the recA-amplicons of the symbionts and analyzed the deletion profiles (Figure 2B). The longest amplicon was that of the symbiont of C. phaseoliformis or C. fossajaponica. They were thus thought to be the most similar to that of the common ancestor of clade I and II symbionts. In clade II symbionts, the nucleotide sequence of the recAamplicon of the C. fossajaponica symbiont was the most similar to that of the $C$. phaseoliformis symbiont, or vice versa. However, the sequence identity of the recA coding region between symbionts of $C$. phaseoliformis and $C$. fossajaponica (90.1\%) was lower than the identities of those of symbionts of C. fausta, C. nautilei and C. pacifica compared with that of the C. phaseoliformis symbiont (95.6\%, 95.5\% and 95.3\%, respectively). To compare the sequences of recA-amplicons, we selected that of the C. phaseoliformis symbiont as a reference.

Compared with the recA-amplicon of the C. phaseoliformis symbiont, that of Rma had several deletions (Figure $2 \mathrm{~B}$ ). Deletions of almost the same size were found in symbionts of C. fausta (Figure 2B; from position 1857 to 2123 in Additional file 1 Figure S1) and of C. pacifica (Figure 2B; from position 1859 to 2125 in Additional file
1 Figure S1), while no such deletion was found in the symbionts of $C$. stearnsii, C. nautilei or C. fossajaponica (Figure 2B).

In clade I symbionts, $r e c A$ was not recognized in their recA-amplicons. However, large deletions of a similar size were found in their respective recA-amplicons (Figure 2B). While some DNA fragments corresponding to the $\mathrm{N}$-terminal RecA remained, $r e c A$ was markedly disintegrated due to the large deletion (Figure 2B).

\section{DNA repair gene, mutY, in Calyptogena clam symbionts}

By performing a BLAST search of unannotated ORFs and pseudogenes in the Rma genome [16], we found that a translated amino acid sequence of Rmag_0017 (21165-22172) was homologous to MutY of other bacteria (48\% identity to that of Alteromonas macleodii str. deep ecotype or $45 \%$ identity to that of Thiomicrospira crunogena XCL-2), but this gene was found to be a pseudogene due to the amber mutation "UGA" at codon 167. No such ORF was recognized in the Vok genome. A primer set, mutY_F and mutY_R, was designed to amplify the genomic region containing mutY (Table 2). The PCR products were designated as mutY-amplicons. An apparently intact mut $Y$ was found in each mutYamplicon of the genomes of clade II symbionts except for Rma, i.e., symbionts of C. phaseoliformis, C. fossajaponica, C. pacifica, C. stearnsii, C. fausta and C. nautilei (Figure $3 \mathrm{~A}$ ), of which the gene product showed significant similarity to MutY of other bacteria. DNA sequence homology analysis with multiple alignment showed that the nucleotide sequences of mutY-amplicons from symbionts in clade II except for Rma showed greater similarity to that of the C. phaseoliformis symbiont (greater than 90\%), while the identity with Rma 


\section{$\operatorname{rec} A$}

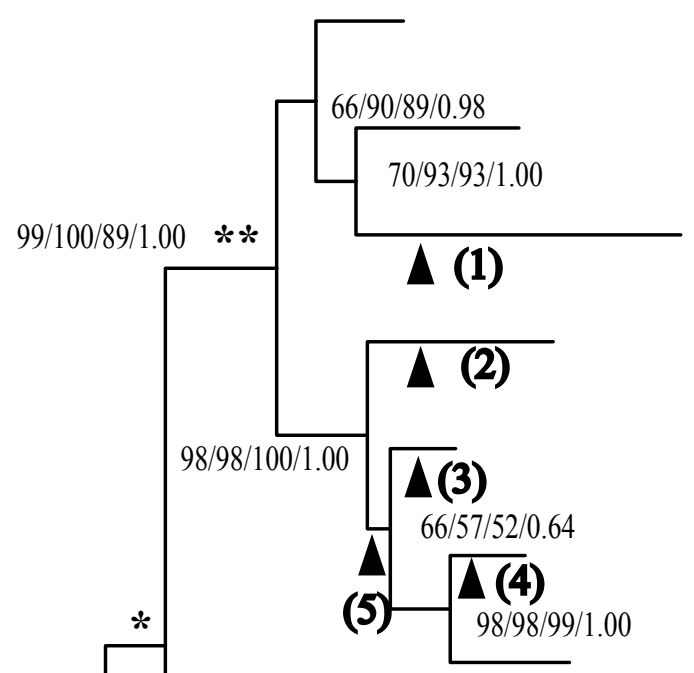

Cpha S

Cfos S
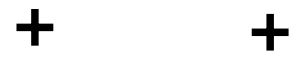

Cfos $\mathrm{S}$
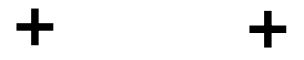

Rma

Cste S

Cnau S

Cfau S

Cpac S

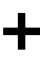

$+$

Csoy S

$99 / 99 / 97 / 1.00$

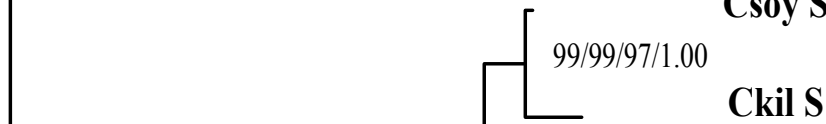

Vok

$100 / 100 / 100 / 1.00$

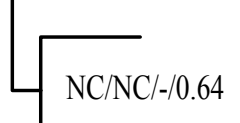

Clau S

Ckaw S

\section{Bsep S}

0.0070

Figure 1 Phylogenetic tree of the Calyptogena clam symbionts with a table showing the presence/absence of mut $Y$ and recA. The maximum likelihood tree based on concatenated 165 and 235 rDNA sequences is shown with bootstrap values (>50\%) obtained from the neighbor joining, maximum parsimony and maximum likelihood methods, and with the posterior probabilities obtained in the Bayesian method at each node. The table shows the presence (+) and absence (-) of functional mutY and recA. Abbreviations of the symbionts and accession numbers of the sequences are shown in Table 3. Black triangle (1), Inactivation of recA by deletions (see Figure $2 \mathrm{~B}$ and Additional file 1, Figure S1), and a substitution mutation of $\mathrm{G}$ to $A$ at position 568 to truncate mutY and deletions at positions 797-799 and 974-989 of mutY in Rma (see Additional file 2, Figure S2). Black triangle (2), Inactivation of recA by substitution and deletion mutations in the C. stearnsii symbiont. Black triangle (3), Inactivation of recA by a two-base insertion of " $\mathrm{CC}$ " at position 483-484, making a new stop codon, in the $C$. nautilei symbiont (see Additional file 1, Figure S1). Black triangle (4), Inactivation of recA by a two-base insertion of "CC" at position 483-484, making a new stop codon, in the C. fausta symbiont lineage (see Additional file 1, Figure S1). Black triangle (5), A deletion of almost the same size outside the recA coding region in the recA-amplicon region in the common ancestor of C. fausta and C. pacifica symbionts (Additional file 1, Figure S1: positions 18552123 for C. fausta symbiont and 1859-2125 for C. pacifica symbiont). Black triangle (6), Occurrence of large-sized deletions in recA- and mutYamplicons in the common ancestor of clade I symbionts (Figure 2).

was lower (80-90\%) (Figure 3B). In addition, two small inframe deletions (3 bp and 15 bp; positions from 797 to 799 and from 974 to 988 in Additional file 2, Figure S2) were found in the nucleotide sequences of Rma
mutY. On the other hand, the mutY-amplicons from clade I symbionts were much shorter than that of the $C$. phaseoliformis symbiont, showing that it was highly degraded by deletions and substitutions (Figure 3B). 

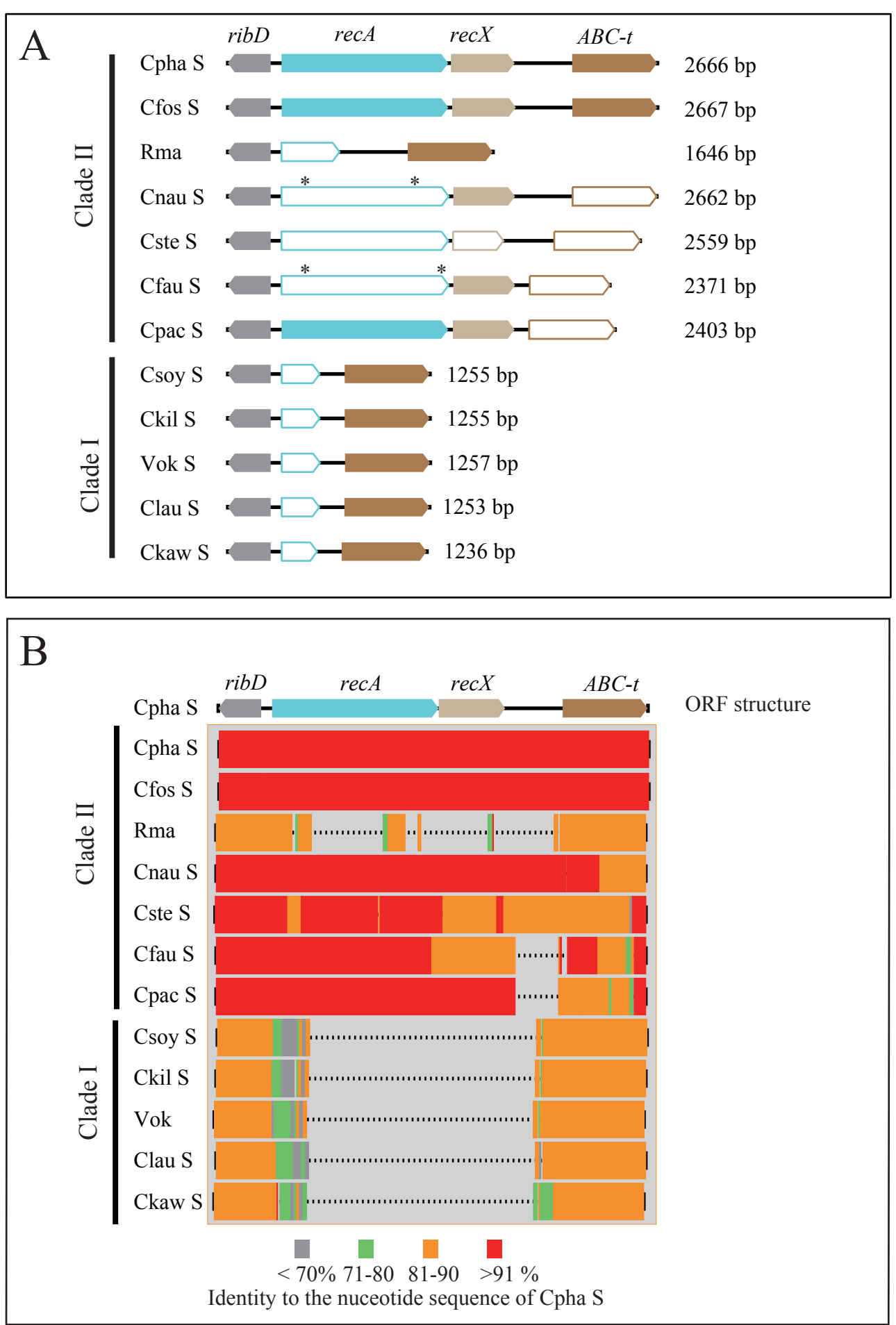

Figure 2 Open reading frames and nucleotide sequence homologies in the recA-amplicons from Calyptogena clam symbionts. A, Open reading frames (ORFs) of the recA-amplicons. Filled column, intact gene. Open columns, pseudogene or remnant gene. Gray column, part of the gene for RibD (bifunctional riboflavin biosynthesis protein). Blue column, recA. Light gray column, rec $X$. Brown column, part of the gene for the ABC transporter ATP-binding protein $(A B C-t) .{ }^{*}$, mutated stop codon. B, Aligned recA-amplicons showing their nucleotide sequence identities and deletion profiles with that of the C. phaseoliformis symbiont. 

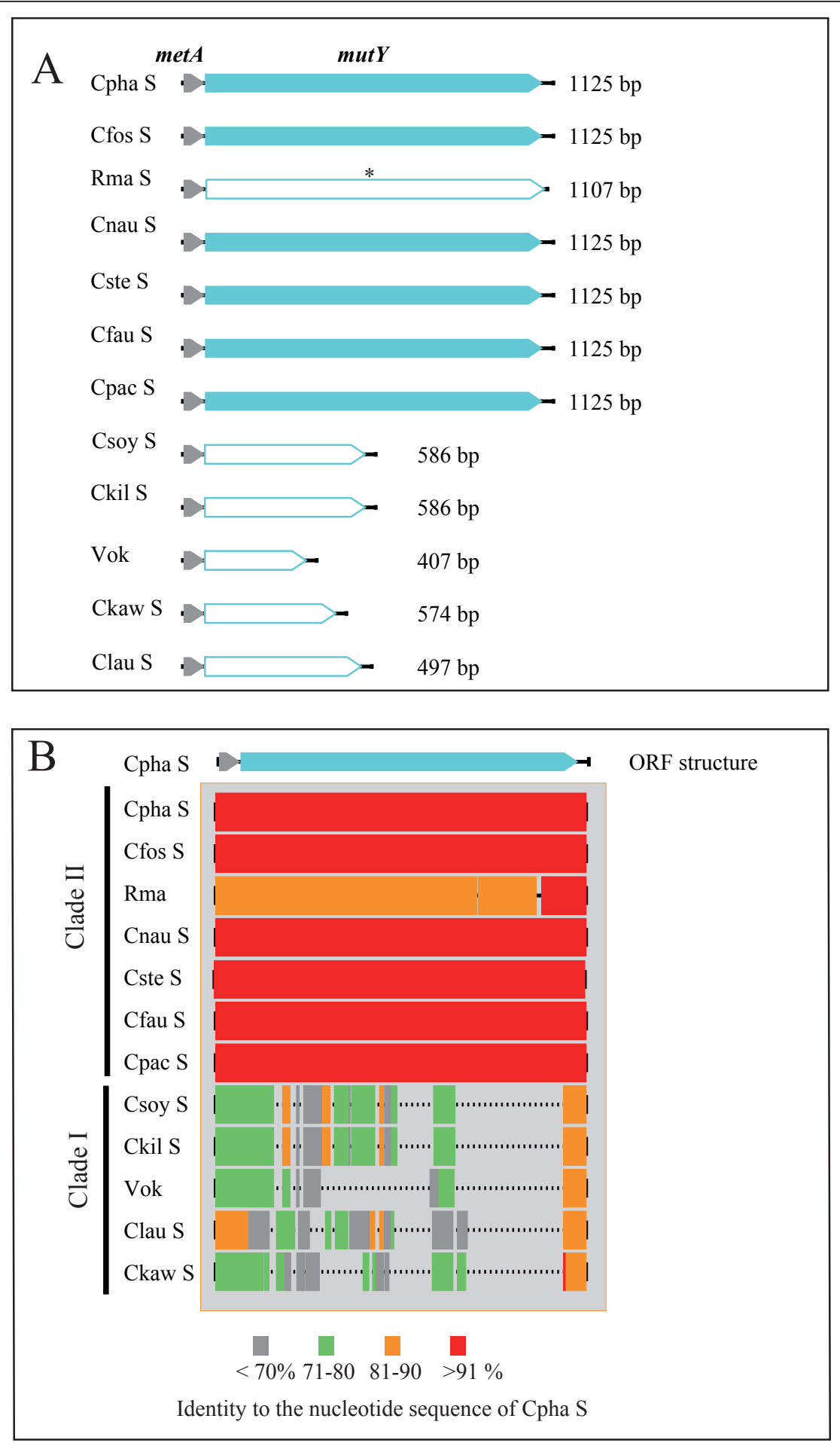

Figure 3 Open reading frames and nucleotide sequence homologies in amplicons for the mut $Y$ region amplified from Calyptogena clam symbiont genomes. A, ORFs in the mutY-amplicons. Filled pentagonal column, intact gene. Open pentagonal column, pseudogene or remnant of gene. Gray, part of metA (homoserine O-succinyltransferase). Blue, mutY. B, Aligned mutY-amplicons showing their nucleotide sequence identities and deletion profiles compared with that of the C. phaseoliformis symbiont. Dotted lines, deletions. 
Because MutY was expected to contribute GC bias to the genomes of the Calyptogena clam symbionts, we analyzed the $\mathrm{G}+\mathrm{C}$ contents of their $16 \mathrm{~S}$ and $23 \mathrm{~S}$ rRNA genes (Table 4). The mean $\mathrm{G}+\mathrm{C}$ content of the $16 \mathrm{~S}$ rRNA genes in clade I symbionts and Rma, which lacked intact mutY, and that in clade II symbionts except for Rma were $49.01 \pm 0.59$ and $50.21 \pm 0.29$ (\%: mean $\pm \mathrm{SD}$ ), respectively. This difference was significant in the $t$-test $(\mathrm{p}=0.00119)$ (Table 4$)$. Those of $23 \mathrm{~S}$ rRNA genes in the symbionts lacking mutY (clade I symbionts and Rma) and in the symbionts with mutY (clade II symbionts except for Rma) were $48.25 \pm 0.35$ and $49.47 \pm 0.36$ (\%: mean \pm SD), respectively, and the difference was significant $(\mathrm{p}=0.000143)$ in the $t$-test. The $\mathrm{G}+\mathrm{C}$ contents of the $16 \mathrm{~S}$ and $23 \mathrm{~S}$ rRNA genes of Rma were $50.20 \%$ and $48.94 \%$, respectively, which were intermediate between those of the symbionts with or without mutY (Table 4). This may indicate that mutY in Rma became a pseudogene relatively recently.

\section{Discussion}

Previously, we reported that $r e c A$ was probably lost in the early stage of RGE in Calyptogena clam symbionts [8]. The present study showed that some of the extant clam symbionts still have intact recA (Figure 2). We hypothesized that in the early phase of RGE of the clam symbionts before the loss of $r e c A$, large-sized deletions occurred due to RecA-dependent recombination [8]. This type of deletion requires repeated sequences larger than $200 \mathrm{bp}$, which have been depleted from the genomes of Rma and Vok $[8,19]$. It is still not clear whether the genomes of the Calyptogena clam symbionts containing recA have large-sized ( $>200 \mathrm{bp}$ ) repeated sequences. The presence of intact or of nearly intact recA and of $m u t Y$ in clade II symbionts except for Rma suggests that the genomes of clade II symbionts are larger than those of clade I symbionts and that their RGE is in an earlier stage than in clade I symbionts. To resolve these questions, we must await their genome sequence analyses.

The coding region of $r e c A$ was shown to be mostly deleted in Rma and clade I symbionts (Figure 2A and 2B). A similar large-sized deletion was found in each of the recA-amplicons of clade I symbionts (Figure 2B). This indicates that the shared part of their deletions occurred in the common ancestor of clade I symbionts after divergence from that of clade II symbionts [arrowhead (6) in Figure 1]. While both Rma and clade I symbionts lack recA, the phylogenetic tree strongly suggests that these losses occurred independently in both the ancestral Rma and the common ancestor of clade I symbionts (Figure 1).

Table 4 Effect of mutY on the GC content of ribosomal RNA genes in Calyptogena clam symbionts

\begin{tabular}{|c|c|c|c|c|c|c|}
\hline \multirow[t]{2}{*}{ Host clam } & \multirow[t]{2}{*}{ Clade } & \multirow[t]{2}{*}{ mutY } & \multicolumn{2}{|c|}{ 16S rDNA } & \multicolumn{2}{|c|}{235 rDNA } \\
\hline & & & Length (bp) & $\mathrm{G}+\mathrm{C} \%$ & Length (bp) & $\mathrm{G}+\mathrm{C} \%$ \\
\hline Calyptogena kilmeri & 1 & - & 1428 & 48.79 & 2519 & 48.14 \\
\hline C. kawamurai & । & - & 1510 & 48.77 & 2519 & 47.94 \\
\hline C. soyoae & । & - & 1508 & 48.77 & 2525 & 48.13 \\
\hline C. laubieri & । & - & 1343 & 48.65 & 2519 & 48.25 \\
\hline C. okutanii & । & - & 1468 & 48.87 & 2525 & 48.12 \\
\hline C. magnifica & $\|$ & - & 1468 & 50.20 & 2536 & 48.94 \\
\hline Mean & I + Rma* & & & 49.01\# & & $48.25 \S$ \\
\hline SD & & & & 0.59 & & 0.35 \\
\hline Mean & I only & & & $48.77 \$$ & & $48.12 !$ \\
\hline SD & & & & 0.08 & & 0.11 \\
\hline C. phaseoliformis & $\|$ & + & 1506 & 50.41 & 2530 & 49.81 \\
\hline C. fossajaponica & $\|$ & + & 1467 & 50.64 & 2531 & 49.99 \\
\hline C. stearnsii & $\|$ & + & 1467 & 49.90 & 2535 & 49.07 \\
\hline C. nautilei & $\|$ & + & 1506 & 49.90 & 2528 & 49.36 \\
\hline C. fausta & $\|$ & + & 1507 & 50.13 & 2534 & 49.31 \\
\hline C. pacifica & $\|$ & + & 1428 & 50.27 & 2532 & 49.25 \\
\hline Mean & II except Rma* & & & $50.21 \# \$$ & & $49.47 \S !$ \\
\hline SD & & & & 0.29 & & 0.36 \\
\hline
\end{tabular}

${ }^{*}$ Ca. Ruthia magnifica (symbiont of C. magnifica)

\#Difference between $\mathrm{G}+\mathrm{C}$ contents of $16 \mathrm{~S}$ rRNA genes of clade I symbionts+Rma and of clade II symbionts except Rma was significant $(p=0.00119)$ in the $t$-test. \$Difference between $\mathrm{G}+\mathrm{C}$ contents of $16 \mathrm{~S}$ rRNA genes of clade I symbionts and of clade II symbionts except Rma $(p=0.00257)$ was significant in Welch's test. §Differences between $\mathrm{G}+\mathrm{C}$ contents of $23 \mathrm{~S}$ rRNA genes of clade I symbionts+Rma and of clade II symbionts $(\mathrm{p}=0.00014)$ was significant in the $t$-test. !Difference between G+C contents of 235 rRNA genes of clade I symbionts and of clade II symbionts $(p=2.014 \mathrm{e}-5)$ was significant in the $t$-test. 
Degradations of the ORFs for $\operatorname{rec} A$ in Rma and in the symbionts of $C$. stearnsii, C. fausta and C. nautilei indicate that RGE in the extant clade II symbionts of Calyptogena clams is in the transitional stage of $r e c A$ loss. The loss of $\operatorname{rec} A$ may start with the degeneration of its ORF by point mutations or a few base insertion/deletion mutations like those in the symbionts of C. fausta, $C$. nautilei and C. stearnsii (Figures 2 and Additional file 2, Figure S1), then continue in the next stage with larger deletions, e.g., those in Rma and in clade I symbionts (Figures 2 and Additional file 2, Figure S1), generated by successive illegitimate recombinations or replication slippages without RecA $[8,23]$. This also suggests that the longer (> $200 \mathrm{bp}$ ) repeated sequences were depleted in the symbiont genome, and that as a result RecA was not able to function as a recombinase or a deletion generator in the genome before losing this gene.

A three-dimensional (3D) homology model of RecA reconstruction using the crystal structure of $E$. coli RecA [24] as a template showed that the 3D structure of RecA in the symbiont of C. phaseoliformis was similar to that of E. coli (Additional files 3 and 4, Figure S3). RecA consists of three domains: the $N$-terminal domain functions as a monomer-monomer interface; the central domain is responsible for ATP binding; and the $C$-terminal domain is responsible for dsDNA binding [24]. This indicates that RecA in the symbionts of C. phaseoliformis, C. fossajaponica and C. pacifica are functional, and that the truncated RecA in C. fausta and C. nautilei symbionts having only the $N$-terminal 68 amino acids is functionless (Additional files 3 and 4, Figure S3).

In the symbiont genomes of C. fausta and C. nautilei, the truncations of their recAs were respectively caused by the same two-base (CC) insertion mutations at the same position of the gene (Additional file 1, Figure S1). It is not clear whether the insertion occurred in the common ancestor of the symbionts of C. fausta, C. nautilei and C. pacifica [arrowhead (5) in Figure 1] and the inserted sequence was removed later in the symbiont of C. pacifica, or whether the insertions occurred independently in the two symbiont lineages of $C$. nautilei and C. fausta [arrowheads (3) and (4) in Figure 1]. If an insertion occurs randomly at any position of the genome, the identical two-base insertion would not likely have occurred independently at the same position of two different genomes at approximately the same time. This question should be addressed in future studies of their genomes.

Because no common insertion/deletion or substitutional mutation making a stop codon was detected among the symbionts of C. stearnsii, C. fausta and $C$. nautilei, the mutations in the $C$. stearnsii symbiont occurred independently in its lineage [arrowhead (2) in Figure 1].
The recAs of C. fausta and C. nautilei symbionts were shown to have additional insertions (Additional file 1, Figure S1). These insertions may have occurred after the loss of the function of the gene by the insertion of "CC" as a result of the relaxation of selective pressure. While RecA is known to be important for recombination and repair mutations, like double-strand breaks of DNA, intracellular symbionts tend to lose it [6]. The selective pressure to retain $\operatorname{rec} A$ probably remained in the early evolutionary stages of the Calyptogena clam symbionts. However, after the loss of large-sized repeated sequences, the selective pressure for retaining $r e c A$ may have decreased.

In clade II symbionts, the present data indicate that their recAs are currently deteriorating. This also supports the above hypothesis that the RGE stage due to recA-dependent deletion is probably ending in these extant genomes.

The DNA repair gene mut $Y$ was found in the genomes of clade II symbionts except for Rma (Figure 1). In Rma, mut $Y$ was found to be split into two ORFs (Figure $3 \mathrm{~A}$ ) by a substitution of the 501 st $\mathrm{G}$ with A, making a new stop codon (Additional file 2, Figure S2). The phylogenetic tree indicates that this mutation occurred in the Rma lineage after divergence from the symbionts of C. phaseoliformis and C. fossajaponica [arrowhead (1) in Figure 1]. MutY has been shown to be composed of the $N$-terminal and $C$-terminal domains (Additional files 5 and 6, Figure S4) [25]. Substrate DNA binds to the cleft between these two domains [26]. While 3D homology modeling showed that MutY of $C$. phaseoliformis, $C$. fossajaponica, C. fausta, C.nautilei and C. pacifica symbionts seemed to have an intact 3D structure and to be functional (Additional files 5 and 6, Figure S4), the split gene products of the Rma mut $Y$ fragments are functionless (Additional files 5 and 6, Figure S4). The evidence that the gene encodes an almost intact amino acid sequence/architecture indicates that Rma lost the functionality of $m u t Y$ relatively recently.

The $\mathrm{G}+\mathrm{C}$ content of genomes generally tends to decrease in obligate intracellular symbionts with decreasing genome size [3]. MutY is known to repair A$\mathrm{G}$ mismatches to $\mathrm{C}-\mathrm{G}$ [20]. The loss of mut $Y$ in a genome is expected to decrease the $G+C$ content $[8,27]$. However, many insect intracellular symbionts such as Buchnera spp. with genomes that have low $\mathrm{G}+\mathrm{C}$ content still have mut $Y$ [8]. In addition, a recently found very small genome of the insect symbiont $\mathrm{Ca}$. Hodgikinia cicadicola lacking mut $Y$ has a high $\mathrm{G}+\mathrm{C}$ content [4]. These may contradict the above view and indicate that the loss of mut $Y$ does not significantly contribute to the decrease in the $\mathrm{G}+\mathrm{C}$ content of the genome. However, in this study, the $\mathrm{G}+\mathrm{C}$ content in the $16 \mathrm{~S}$ and $23 \mathrm{~S}$ rRNA gene sequences was significantly lower in the 
Calyptogena symbionts without mut $Y$ than that in the symbionts with mutY (Table 4). This supports the hypothesis that the loss of mut $Y$ contributes to the GC bias of the genome [20,27]. The G+C content of Rma was intermediate between the two symbiont clades. This agrees with the view that it lost functional mutY more recently than clade I symbionts during evolution. This result also coincides with the data showing that the $G$ $+C$ content of the Rma genome $(34.0 \%)$ is higher than that of Vok (31.6\%) [16,17]. Stewart et al. have recently reported that the $\mathrm{G}+\mathrm{C}$ contents of 9 genes including $16 \mathrm{~S}$ and 23S RNA genes of the symbionts in the gigas/ kilmeri clade that corresponds to clade I in the present study were significantly lower than those of another clade that corresponds to clade II in the present study (Additional file 7, Figure S5) [28]. Although it was not clear whether the symbionts in the other clade reported by Stewart et al. [28] had mutY or not, the present results suggest that they do and thus the $\mathrm{G}+\mathrm{C}$ contents of their genes are higher than those of the symbionts in the gigas/kilmeri clade.

It has recently been shown that mutational bias of $\mathrm{GC} \rightarrow \mathrm{AT}$ is a general trend in bacteria, and this trend may be counterbalanced by biased gene conversion and natural selection to maintain the $G+C$ contents [29-31]. In intracellular symbionts, relaxation of natural selection, lower recombination frequency, small effective population size, codon usage, availability of nucleotides in the cytoplasmic pool and loss of DNA repair genes may contribute to lower $\mathrm{G}+\mathrm{C}$ content $[4,31,32]$. In addition to the loss of $\operatorname{mut} Y$, any of these factors may have also contributed to a greater reduction of the $\mathrm{G}+\mathrm{C}$ content in symbionts in clade I compared with those in clade II. However, this remains to be studied in future.

The present phylogenetic tree shows that both mutY and $\operatorname{rec} A$ have been lost in Rma and in clade I symbionts (Figure 1). Were the losses in clade I symbionts and Rma accidental coincidences or related phenomena? The loss of $r e c A$ may increase the mutation rate of the genome and hence increase the possibility of losing other genes such as mutY. It is also noteworthy that the branch length of Rma is longer than other branches in the clade II lineage, and the branch length from the node between clade I and II symbionts (* in Figure 1) to the node of clade I symbiont radiation ( $* *$ in Figure 1 ) is longer than the length to the node of clade II symbiont radiation (** in Figure 1). As a result, the loss of $\operatorname{rec} A$ which occurred in Rma and clade I symbionts independently may have increased the mutation rate and elongated these branch lengths. This may also increase the probability of losing other genes including mutY.

Once genes lose their functions, their selective pressure must be relaxed and their mutation rates are expected to increase [33]. In the functionless $r e c A s$ of $C$. fausta and $C$. nautilei, one additional mutation was found in each (Additional file 1, Figure S1). Two additional deletions were also found in the Rma mutY (Additional file 2, Figure S2). These may be the result of the decreased (relaxed) selective pressure after the losses of the functions of the genes.

While an evolutionary event like the loss of a gene for DNA repair or recombination may occur spontaneously in a certain lineage, it must greatly affect the later evolutionary fate of that lineage. We previously suggested that the loss of $r e c A$ probably stabilized the genome architecture in Calyptogena clam symbionts $[8,34]$. The present data raise the possibility that the loss of mut $Y$ affected the $\mathrm{G}+\mathrm{C}$ content of the genomes of the Calyptogena symbionts. The effect of losing genes for DNA recombination and repair on their RGE will be analyzed by sequencing the genomes of other Calyptogena clam symbionts, which is now in progress and will be published elsewhere.

\section{Conclusion}

The apparently intact genes for DNA recombination and repair recA and mut $Y$ were found in some clade II symbionts, i.e., symbionts of C. phaseoliformis, C. fossajaponica and C. pacifica. Those of C. stearnsii, C. nautilei and $C$. fausta had intact mut $Y$ but their recA was found to be a pseudogene due to insertion/deletion and/or substitution mutations. These genes were disintegrated and lost in Rma and in clade I symbionts. Most of the rec $A$ coding region was lost in the common ancestor of clade I symbionts and in the Rma lineage as a result of deletions. In the symbionts of C. stearnsii, C. fausta and C. nautilei, recA became functionless due to small base insertions and substitutions. The mut $Y$ gene of Rma was disintegrated by a substitutional mutation. mut $Y$ was also lost through deletions in the common ancestor of clade I symbionts. The coinciding losses of both recA and mut $Y$ in Rma and clade I symbionts are thought to have occurred independently in the respective lineages.

The $\mathrm{G}+\mathrm{C}$ contents of the symbionts with mut $Y$ were significantly higher than in those without mutY. This indicates that the loss of mut $Y$ probably decreased the $\mathrm{G}+\mathrm{C}$ contents of the descendant symbiont genomes. This suggests that gene degradation, which occurs by chance in some lineages of symbionts, greatly affects the genomes of later descendants of the lineage.

\section{Methods}

\section{Sample collection}

Calyptogena clams were collected and stored at $-80^{\circ} \mathrm{C}$ in a freezer until use (Table 1). C. pacifica, C. stearnsii and C. kilmeri were collected in Monterey Bay (Table 1) and were kind gifts to JAMSTEC from Dr. J. Barry of the Monterey Bay Aquarium Research Institute. 


\section{DNA extraction}

The gill tissue was dissected, washed with filtered (0.2$\mu \mathrm{m}$ pore membrane filter; Millipore), sterilized artificial seawater to remove bacteria attached to the gill surface and chopped with scissors. DNA was extracted from approximately $10 \mathrm{mg}$ of the tissue with a DNeasy Tissue Kit (Qiagen) according to the manufacturer's instructions. Although some bacteria attached to the surface of the gill tissue might have remained after washing, only the amplified products of the symbionts were detected in PCR for the 16S rRNA gene. This indicated that bacteria contaminating the surface of the gills were far less numerous than the symbionts.

\section{Primer design}

Almost whole-length genes for the small subunit ribosomal RNA (16S rRNA) and the large subunit ribosomal RNA (23S rRNA) were amplified from 10 Calyptogena clam symbionts with specific primers (Table 2).

Based on the conserved RecA amino acid sequences of several gamma-proteobacteria, a set of internal consensus primers was designed for PCR amplification of recA in the Calyptogena clam symbionts (Table 2). In PCR, a DNA fragment amplified from C. phaseoliformis symbiont DNA was detected. A BLAST search showed that the DNA fragment obtained was a portion of recA. We then searched for $r e c A$ or its remnant in the genomes of Rma and Vok with BLASTX using recA in E. coli str. 12, substr. MG 1655 (accession number $=$ NC_000913, gene locus tag $=\mathrm{b} 2699$ ) as a reference and found a remnant gene sequence of $r e c A$ in the Rma genome. A set of primers was designed from the conserved franking regions of the corresponding regions of the Rma and Vok genomes. The primers were designated as the external primers for $r e c A$ (Table 2). Primers for PCR of mut $Y$ were designed based on the franking regions of the remnant sequences of mut $Y$ detected in the Rma genome and their corresponding sequences in Vok (Table 2).

\section{Amplification of the ribosomal RNA genes, recA and mutY, from Calyptogena clam symbionts}

The genome regions containing $16 S$ rRNA, $23 S$ rRNA, mut $Y$, recA or their corresponding regions were amplified by PCR with the primer sets shown in Table 2 . According to the manufacturer's instructions, the reaction mixture contained $1 \mu \mathrm{l}$ of template solution containing $100 \mathrm{ng}$ of DNA, $5 \mu \mathrm{l}$ of $10 \times$ ExTaq buffer (Takara), $4 \mu \mathrm{l}$ of dNTP mix (Takara), $1 \mu \mathrm{l}$ of the 10 $\mathrm{pmol} / \mu \mathrm{l}$ forward primer solution, $1 \mu \mathrm{l}$ of the $10 \mathrm{pmol} / \mu \mathrm{l}$ reverse primer solution, $0.25 \mu \mathrm{l}$ of ExTaq polymerase solution (Takara) and $37.75 \mu \mathrm{l}$ of pure water. The reaction mixture was initially incubated at $96^{\circ} \mathrm{C}$ for $2 \mathrm{~min}$, then subjected to 35 cycles of the PCR protocol $\left(96^{\circ} \mathrm{C}\right.$ for $20 \mathrm{~s}, 55^{\circ} \mathrm{C}$ for $45 \mathrm{~s}$ and $72^{\circ} \mathrm{C}$ for $3 \mathrm{~min}$ ) and finally to extension at $72^{\circ} \mathrm{C}$ for 10 min with a Takara TP600 Thermal cycler. The reaction mixture $(2 \mu \mathrm{l})$ was applied to $1 \%$ agarose gel electrophoresis to check the amplicons. The gel was stained with $0.6 \%$ ethidium bromide solution to visualize the amplicon bands. The amplified DNA was purified with a Wizard SV Gel and a PCR Clean-Up System Kit (Promega) according to the manufacturer's instructions.

The nucleotide sequences of the amplified and purified DNAs were determined using a Big Dye Terminator v3.1 Cycle Sequencing Kit (Applied Biosystems) and an ABI PRIZM 3100 Genetic Analyzer (Applied Biosystems) according to the manufacturer's instructions. The sequences obtained were submitted to DDBJ-EMBLGENBANK, and their accession numbers are listed in Table 3.

Phylogenetic relationships of Calyptogena clam symbionts were analyzed using the genes for $16 \mathrm{~S}$ rRNA and $23 \mathrm{~S}$ rRNA, some of which were retrieved from DDBJEMBL-GENBANK. The $16 \mathrm{~S}$ and $23 \mathrm{~S}$ rRNA gene sequences were concatenated and aligned using MAFFT $6[34,35]$. The alignment obtained was manually refined, and ambiguous nucleotide positions were excluded using Se-Al ver. 2.0all [36]. The aligned sequences (3128 $\mathrm{bp})$ were analyzed using the neighbor-joining $(\mathrm{NJ})$ and maximum composite likelihood methods [37] and maximum parsimony (MP) methods with MEGA 4 [38,39], as well as with the maximum likelihood (ML) method with PAUP* 4.0 [39]. The NJ tree was constructed with the maximum composite likelihood method distance [37]. MP analysis was performed with a heuristic search using close neighbor interchange (level $=1$ ), a branchswapping method with initial trees generated by random addition (10 replications); a complete deletion option was used to treat gaps/missing data. Modeltest ver 3.7 [40] was used to select the appropriate model of evolution for the ML analysis, with the Akaike information criterion. ML analysis was performed using the GTR $+\mathrm{I}$ + G model [41], and optimized parameter values were applied after the determination using Modeltest. The reliability of the tree topology was assessed by bootstrap resampling (number of pseudoreplicates: NJ and MP, 1000; ML, 100). They were also analyzed by the Bayesian method using MrBayes 3.1 [42]. The posterior probabilities were calculated to assess the reliability of the tree topology. For Bayesian analysis, we determined the optimal model of sequence evolution for each of the two genes (16S rRNA and 23S rRNA genes) using MrModeltest 2.2 [43]. The GTR+I+G model was selected for both the 16S rRNA and 23S rRNA genes. Bayesian analysis was performed with random starting trees and unlinked parameters and run for 5,000,000 generations, sampling the Markov chains at intervals of 
100 generations. Four heated Markov chains (using default heating values) were used. The first 12,500 of the 50,000 resulting trees were discarded as "burn-in." To ensure that Markov chains were not trapped on local optima, Bayesian inferences were performed twice, beginning with different starting trees, and apparent stationary levels were compared for convergence [44].

To locate recA and mut $Y$ in the amplified DNA fragments, a search was performed for an ORF, and then a BLAST search against the NCBI protein database (nr) was performed. Multiple alignments for their nucleotide sequences were constructed using the Multi-LAGAN program [45].

\section{Additional material}

Additional file 1: Figure S1. Multiple sequence alignments of recAamplicons from Calyptogena clam symbiont genomes. The recA-

containing genome region (recA-amplicon) was amplified with a primer set [recA_F (5'-GATTGCATATCATTCATCTGATAACG-3'), recA_R (5'-

AGTGGATTRGGATCAAGCATAGC-3')] from 9 Calyptogena clam symbionts using the PCR and from 2 symbiont genomes [Vesicomyosocius okutanii (Vok: accession \# = AP009247) and Ruthia magnifica (Rma: accession \# = CP000488)] in in silico PCR. Abbreviations of symbionts are shown in Table 3. Gray background-colored horizontal sequence in Cpha S, part of ribD; blue background-colored horizontal sequence in Cpha S, recA; light gray background horizontal sequence in $\mathrm{Cpha} S$, rec $X_{\text {; }}$ brown background horizontal sequence of Cpha S, part of ABC-t (ABC transporter ATPbinding protein gene). *Identical nucleotide in the aligned sequences. Gray background vertical column, gap in Cpha S sequence. Red letters, in-frame start codon of recA or mutated recA ORFs. Blue letters, in-frame stop codon.

Additional file 2: Figure S2. Multiple sequence alignment of mutYamplicons from Calyptogena clam symbiont genomes. The mutYcontaining genome region (mutY-amplicon) was amplified with a primer set [mutY_F and mutY_R (Table 2)] from 9 Calyptogena clam symbionts in PCR and from 2 symbiont genomes [Vesicomyosocius okutanii (Vok: accession \# = AP009247) and Ruthia magnifica (Rma: accession \# = (P000488)] in in silico PCR. Abbreviations of symbionts are shown in Table 3. Gray background horizontal sequence in Cpha S, part of metA (homoserine O-succinyltransferase); blue background sequence in Cpha $\mathrm{S}$, mutY. ${ }^{*}$ Identical nucleotide in the aligned sequences. Gray vertical column, gap in Cpha S sequence. Red letters, in-frame start codon of the original or mutated mutY ORFs. Blue letters, in-frame stop codon.

Additional file 3: Figure S3. Part A. 3D homology models reconstructed for RecA of the Calyptogena phaseoliformis symbiont. Homology modeling using the Swiss-Model Workspace (http:// swissmodel.expasy.org/) was based on the 3D structure of Escherichia coli RecA (PDB accession number 1U94: [24]) as a template. A, Alignment of amino acid sequences of Calyptogena clam symbiont RecA and E. coli. RecA. Sequences were aligned with ClustalW. a, RecA sequence from Lys6 to Pro-331 in E. coli; b, secondary structure of E. coli RecA (1U94). Red rectangles, $\alpha$-helices; blue arrows, $\beta$-strands. C, RecA sequence from Lys- 5 to Thr-329 in the symbiont of C. phaseoliformis. d, RecA sequence of $\mathrm{N}$ terminal ORF in the symbiont of $C$. fausta. e, RecA sequence of $C$ terminal ORF in the symbiont of $C$. fausta.

\section{Additional file 4: Figure S3. Parts B-E. 3D homology models} reconstructed for RecA of the Calyptogena phaseoliformis symbiont. Homology modeling using the Swiss-Model Workspace (http:// swissmodel.expasy.org/) was based on the 3D structure of Escherichia coli RecA (PDB accession number 1U94: [24]) as a template. B, Homology model reconstructed for C. phaeoliformis symbiont RecA. C, Homology model reconstructed for $\mathrm{N}$-terminal and $\mathrm{C}$-terminal amino acid peptides of RecA in C. fausta symbiont. $\mathrm{N}$-terminal and $\mathrm{C}$-terminal peptides are shown in violet and light blue, respectively. D, Crystal structure of E. coli
RecA (accession \# = 1U94); $\alpha$-helices and $\beta$-sheets are indicated in redgreen, and light blue, respectively. E, Merged 3D structures of RecAs of $E$. coli (D) and of C. phaseoliformis symbionts (B) showing that their 3D structures are nearly the same. This suggests that the C. phaseoliformis symbiont RecA is intact and functional.

Additional file 5: Figure S4. Part A. 3D homology models reconstructed for MutY of the C. phaseoliformis symbiont. The model was reconstructed using the Swiss-Model Workspace (http:// swissmodel.expasy.org/) based on the crystal structure of Geobacillus stearothermophilus MutY [26] (PDB accession \# = 3FSP) as a template. A, Alignment of MutY amino acid sequences of Calyptogena symbionts and G. stearothermophilus. Sequences were aligned using ClustalW. a, MutY sequence from Phe-8 to Ser-360 in G. stearothermophilus. b, Secondary structure of $G$. stearothermophilus MutY (3FSP). Red rectangles, $\alpha$-helices; blue arrows, $\beta$-strands. c, MutY sequence from Val- 1 to Asp-341 in the symbiont of $C$. phaseoliformis. d, MutY sequence of $\mathrm{N}$-terminal ORF in Ruthia magnifica. e, MutY sequence of $C$-terminal ORF in $R$. magnifica.

Additional file 6: Figure S4. Parts B-E. 3D homology models reconstructed for MutY of the C. phaseoliformis symbiont. B, Homology model reconstructed for C. phaseoliformis symbiont MutY. C, Homology model reconstructed for $\mathrm{N}$-terminal (violet) and $\mathrm{C}$-terminal (light blue) amino acid peptides of MutY in R. magnifica. D, Crystal structure of G. stearothermophilus MutY (accession \# = 3FSP). $\alpha$-Helices and $\beta$-sheets are indicated as red-green and light blue, respectively. $E$, Merged 3D structures of MutY of G. stearothermophilus (D) and C. phaseoliformis symbionts (B).

Additional file 7: Figure S5. Phylogenetic tree of the Calyptogena clam symbionts including those of reported in Stewart et al. 2009 [28]. 165 and $23 \mathrm{~S}$ rRNA gene sequences of the symbionts reported in the present study and of those reported in Stewart et al. (2009) [28] were concatenated and used for phylogenetic tree reconstruction. Topology of the tree constructed using the maximum likelihood method is shown with bootstrap values (> 50\%) obtained from the neighbor joining and maximum likelihood methods at each node. Accession numbers of the sequences are shown in the tree. Names and abbreviations of the symbionts are the same as those in Table 3 of the present study or those in [28]. Abbreviations for generic names: C., Calyptogena; $E$., Ectenagena; V., Vesicomya. ${ }^{*}$ Symbionts reported in Stewart et al. [28]; ** symbionts reported in both Stewart et al. [28] and the present study.

\section{Acknowledgements}

James Barry of Monterey-Bay Aquarium Research Institute is acknowledged for donating the samples of Calyptogena pacifica, C. stearnsii and C. kilmeri. We would like to thank Chiaki Kato and Takako Satoh for the sample of $C$. nautilei. Katsunori Fujikura and Yoshihiro Fujiwara are acknowledged, respectively, for the samples of C. fausta and C. kawamurai. We are grateful to Fumio Inagaki, who was the principal investigator of the JAMSTEC RN Yokosuka cruise YK06-05 during which we collected C. phaseoliformis and C. fossajaponica. The captains and crews of cruise YK06-05 are thanked for collecting the biological samples and data. We would like to thank Kiyotaka Takishita for his critical comments on the manuscript. We would also like to thank the anonymous reviewers for their valuable comments on the manuscript.

\section{Author details}

${ }^{1}$ Marine Biodiversity Research Program, Japan Agency for Marine-Earth Science and Technology, Natsushima-cho, Yokosuka, Kanagawa 237-0061, Japan. ${ }^{2}$ Department of Biological Sciences, Graduate School of Science, The University of Tokyo, Hongo, Bunkyo-ku, Tokyo 113-0033, Japan. ${ }^{3}$ Graduate School of Marine Science and Technology, Tokyo University of Marine Science and Technology, Konan, Minato-ku, Tokyo 108-8477, Japan.

\section{Authors' contributions}

The experimental plan and story of this research were designed and organized by TdM. HK and SS performed the experiments and conducted resequencing to confirm the sequences. The phylogenetic analysis was carried out by TrM. DNA sequence data were analyzed by YT. TY 
reconstructed 3D homology models of the gene products. TK contributed to the discussion in this paper. All the data and analyzed results were examined carefully and discussed by all the authors. The final manuscript was read and approved by all the authors.

Received: 11 July 2011 Accepted: 3 October 2011

Published: 3 October 2011

\section{References}

1. Moran NA, Mira A: The process of genome shrinkage in the obligate symbiont Buchnera aphidicola. Genome Biology 2001, 2(12), research/ 10054.1-12.

2. Mira A, Ochman H, Moran NA: Deletional bias and the evolution of bacterial genomes. Trends Genet 2001, 17(10):589-596.

3. Moran NA: Tracing the evolution of gene loss in obligate bacterial symbionts. Current Opinion in Microbiology 2003, 6(5):512-518.

4. McCutcheon JP, McDonald BR, Moran NA: Origin of an alternative genetic code in the extremely small and GC-rich genome of a bacterial symbiont. Plos Genetics 2009, 5(7):e1000565.

5. Rocha EPC, Cornet E, Michel B: Comparative and evolutionary analysis of the bacterial homologous recombination systems. Plos Genetics 2005, 1(2):247-259.

6. Sharples GJ: For absent friends: life without recombination in mutualistic gamma-proteobacteria. Trends Microbiol 2009, 17(6):233-242.

7. Tamas I, Klasson L, Canback B, Naslund AK, Eriksson AS, Wernegreen JJ, Sandstrom JP, Moran NA, Andersson SG: 50 million years of genomic stasis in endosymbiotic bacteria. Science 2002, 296(5577):2376-2379.

8. Kuwahara H, Takaki Y, Yoshida T, Shimamura S, Takishita K, Reimer JD, Kato C, Maruyama T: Reductive genome evolution in chemoautotrophic intracellular symbionts of deep-sea Calyptogena clams. Extremophiles 2008, 12(3):365-374

9. Moran NA, McLaughlin HJ, Sorek R: The dynamics and time scale of ongoing genomic erosion in symbiotic bacteria. Science 2009, 323(5912):379-382

10. Stewart FJ, Newton IL, Cavanaugh CM: Chemosynthetic endosymbioses: adaptations to oxic-anoxic interfaces. Trends Microbiol 2005, 13(9):439-448.

11. Endow K, Ohta S: Occurrence of Bacteria in the Primary Oocytes of Vesicomyid Clam Calyptogena-Soyoae. Marine Ecology-Progress Series 1990, 64(3):309-311.

12. Cary SC, Giovannoni SJ: Transovarial Inheritance of Endosymbiotic Bacteria in Clams Inhabiting Deep-Sea Hydrothermal Vents and Cold Seeps. Proceedings of the National Academy of Sciences of the United States of America 1993, 90(12):5695-5699.

13. Peek AS, Feldman RA, Lutz RA, Vrijenhoek RC: Cospeciation of chemoautotrophic bacteria and deep sea clams. Proc Natl Acad Sci USA 1998, 95(17):9962-9966.

14. Stewart FJ, Young CR, Cavanaugh CM: Lateral symbiont acquisition in a maternally transmitted chemosynthetic clam endosymbiosis. Mol Biol Evol 2008, 25(4):673-687.

15. Okutani T, Koshi-ishi T, Sato T, Imai T, Kato C: Vesicomyid Fauna in the Chishima (Kurile) Trench: Occurrences of a New Taxon and Calyptogena extenta. VENUS 2009, 68(1-2):15-25.

16. Newton IL, Woyke T, Auchtung TA, Dilly GF, Dutton RJ, Fisher MC, Fontanez KM, Lau E, Stewart FJ, Richardson PM, et al: The Calyptogena magnifica chemoautotrophic symbiont genome. Science 2007, 315(5814):998-1000.

17. Kuwahara H, Yoshida T, Takaki Y, Shimamura S, Nishi S, Harada M, Matsuyama K, Takishita K, Kawato M, Uematsu K, et al: Reduced genome of the thioautotrophic intracellular symbiont in a deep-sea clam, Calyptogena okutanii. Curr Biol 2007, 17(10):881-886.

18. Kowalczykowski SC, Dixon DA, Eggleston AK, Lauder SD, Rehrauer WM: Biochemistry of homologous recombination in Escherichia coli. Microbiol Rev 1994, 58(3):401-465.

19. Lovett ST: Encoded errors: mutations and rearrangements mediated by misalignment at repetitive DNA sequences. Molecular Microbiology 2004, 52(5):1243-1253.

20. Au KG, Clark S, Miller JH, Modrich P: Escherichia coli mutY gene encodes an adenine glycosylase active on G-A mispairs. Proc Natl Acad Sci USA 1989, 86(22):8877-8881.
21. Dale C, Wang B, Moran N, Ochman H: Loss of DNA recombinational repair enzymes in the initial stages of genome degeneration. Mol Biol Evol 2003, 20(8):1188-1194

22. Nilsson Al, Koskiniemi S, Eriksson S, Kugelberg E, Hinton JC, Andersson Dl: Bacterial genome size reduction by experimental evolution. Proc Natl Acad Sci USA 2005, 102(34):12112-12116.

23. Rocha EPC: An appraisal of the potential for illegitimate recombination in bacterial genomes and its consequences: From duplications to genome reduction. Genome Research 2003, 13(6):1123-1132.

24. Xing X, Bell CE: Crystal structures of Escherichia coli RecA in a compressed helical filament. J Mol Biol 2004, 342(5):1471-1485.

25. Guan Y, Manuel RC, Arvai AS, Parikh SS, Mol CD, Miller JH, Lloyd S, Tainer JA: MutY catalytic core, mutant and bound adenine structures define specificity for DNA repair enzyme superfamily. Nat Struct Biol 1998, 5(12):1058-1064.

26. Fromme JC, Banerjee A, Huang SJ, Verdine GL: Structural basis for removal of adenine mispaired with 8-oxoguanine by MutY adenine DNA glycosylase. Nature 2004, 427(6975):652-656.

27. Lind PA, Andersson DI: Whole-genome mutational biases in bacteria. Proc Natl Acad Sci USA 2008, 105(46):17878-17883.

28. Stewart FJ, Young CR, Cavanaugh CM: Evidence for homologous recombination in intracellular chemosynthetic clam symbionts. Mol Biol Evol 2009, 26(6):1391-1404.

29. Hershberg R, Petrov DA: Evidence that mutation is universally biased towards AT in bacteria. PLoS Genet 2010, 6(9):e1001115.

30. Hildebrand F, Meyer A, Eyre-Walker A: Evidence of selection upon genomic GC-content in bacteria. PLoS Genet 2010, 6(9):e1001107.

31. Rocha EP, Feil EJ: Mutational patterns cannot explain genome composition: Are there any neutral sites in the genomes of bacteria? PLoS Genet 2010, 6(9):e1001104.

32. Moran NA: Accelerated evolution and Muller's rachet in endosymbiotic bacteria. Proc Natl Acad Sci USA 1996, 93(7):2873-2878.

33. Kimura M: The neutral theory of molecular evolution. Cambridge University Press; 1983.

34. Katoh K, Misawa K, Kuma K, Miyata T: MAFFT: a novel method for rapid multiple sequence alignment based on fast Fourier transform. Nucleic Acids Res 2002, 30(14):3059-3066.

35. Katoh $\mathrm{K}$, Toh H: Improved accuracy of multiple ncRNA alignment by incorporating structural information into a MAFFT-based framework. BMC Bioinformatics 2008, 9:212.

36. , Sequence Alignment Editor v2.0 [http://iubio.bio.indiana.edu/soft/ iubionew/molbio/dna/analysis/Pist/main.html.

37. Tamura $\mathrm{K}, \mathrm{Nei} \mathrm{M}, \mathrm{Kumar} \mathrm{S}$ : Prospects for inferring very large phylogenies by using the neighbor-joining method. Proc Natl Acad Sci USA 2004, 101(30):11030-11035.

38. Tamura K, Dudley J, Nei M, Kumar S: MEGA4: Molecular Evolutionary Genetics Analysis (MEGA) software version 4.0. Mol Biol Evol 2007, 24(8):1596-1599

39. Swofford DL: PAUP*: Phylogenetic analysis using parsimony (and other methods) 4.0 beta. 2002

40. Posada D, Crandall KA: MODELTEST: testing the model of DNA substitution. Bioinformatics 1998, 14(9):817-818.

41. Rodriguez F, Oliver JL, Marin A, Medina JR: The general stochastic model of nucleotide substitution. J Theor Biol 1990, 142(4):485-501.

42. Ronquist F, Huelsenbeck JP: MrBayes 3: Bayesian phylogenetic inference under mixed models. Bioinformatics 2003, 19(12):1572-1574.

43. Nylander JAA: 2004, MrModeltest v2.

44. Huelsenbeck JP, Ronquist F, Nielsen R, Bollback JP: Bayesian inference of phylogeny and its impact on evolutionary biology. Science 2001, 294(5550):2310-2314.

45. Brudno M, Do CB, Cooper GM, Kim MF, Davydov E, Green ED, Sidow A, Batzoglou S: LAGAN and Multi-LAGAN: efficient tools for large-scale multiple alignment of genomic DNA. Genome Res 2003, 13(4):721-731.

doi:10.1186/1471-2148-11-285

Cite this article as: Kuwahara et al:: Loss of genes for DNA recombination and repair in the reductive genome evolution of thioautotrophic symbionts of Calyptogena clams. BMC Evolutionary Biology 2011 11:285. 